Undergraduate Texts in Mathematics

Editors

S. Axler

F.W. Gehring

P.R. Halmos

Springer Science+Business Media, LLC 
Anglin: Mathematics: A Concise History and Philosophy.

Readings in Mathematics.

Anglin/Lambek: The Heritage of Thales.

Readings in Mathematics.

Apostol: Introduction to Analytic Number Theory. Second edition.

Armstrong: Basic Topology.

Armstrong: Groups and Symmetry.

Axler: Linear Algebra Done Right.

Bak/Newman: Complex Analysis. Second edition.

Banchoff/Wermer: Linear Algebra Through Geometry. Second edition.

Berberian: A First Course in Real Analysis.

Brémaud: An Introduction to Probabilistic Modeling.

Bressoud: Factorization and Primality Testing.

Bressoud: Second Year Calculus. Readings in Mathematics.

Brickman: Mathematical Introduction to Linear Programming and Game Theory.

Browder: Mathematical Analysis: An Introduction.

Buskes/van Rooij: Topological Spaces: From Distance to Neighborhood.

Cederberg: A Course in Modern Geometries.

Childs: A Concrete Introduction to Higher Algebra. Second edition.

Chung: Elementary Probability Theory with Stochastic Processes. Third edition.

Cox/Little/O'Shea: Ideals, Varieties, and Algorithms. Second edition.

Croom: Basic Concepts of Algebraic Topology.

Curtis: Linear Algebra: An Introductory Approach. Fourth edition.

Devlin: The Joy of Sets: Fundamentals of Contemporary Set Theory. Second edition.
Dixmier: General Topology.

Driver: Why Math?

Ebbinghaus/Flum/Thomas:

Mathematical Logic. Second edition.

Edgar: Measure, Topology, and Fractal

Geometry.

Elaydi: Introduction to Difference Equations.

Exner: An Accompaniment to Higher Mathematics.

Fine/Rosenberger: The Fundamental Theory of Algebra.

Fischer: Intermediate Real Analysis.

Flanigan/Kazdan: Calculus Two: Linear and Nonlinear Functions. Second edition.

Fleming: Functions of Several Variables. Second edition.

Foulds: Combinatorial Optimization for Undergraduates.

Foulds: Optimization Techniques: An Introduction.

Franklin: Methods of Mathematical Economics.

Gordon: Discrete Probability.

Hairer/Wanner: Analysis by Its History. Readings in Mathematics.

Halmos: Finite-Dimensional Vector Spaces. Second edition.

Halmos: Naive Set Theory.

Hämmerlin/Hoffmann: Numerical Mathematics.

Readings in Mathematics.

Hijab: Introduction to Calculus and Classical Analysis.

Hilton/Holton/Pedersen: Mathematical Reflections: In a Room with Many Mirrors.

Iooss/Joseph: Elementary Stability and Bifurcation Theory. Second edition.

Isaac: The Pleasures of Probability. Readings in Mathematics.

James: Topological and Uniform Spaces.

Jänich: Linear Algebra.

Jänich: Topology.

(continued after index) 
Pierre Brémaud

\section{An Introduction to Probabilistic Modeling}

With 90 Illustrations 
Pierre Brémaud

Laboratoire des Signaux et Systèmes

CNRS

Plateau de Moulon

F91192 Gif-sur-Yvette

France

Editorial Board

S. Axler

Department of Mathematics

Michigan State University

East Lansing, MI 48824

USA

F.W. Gehring

Department of Mathematics

University of Michigan

Ann Arbor, MI 48019

USA
P.R. Halmos

Department of Mathematics

Santa Clara University

Santa Clara, CA 95053

USA

Mathematics Subject Classification (1980): 60-01

Library of Congress Cataloging-in-Publication Data

Brémaud. Pierre.

An introduction to probabilistic modeling.

(Undergraduate texts in mathematics)

Bibliography: $p$.

Includes index.

1. Probabilities. I. Title. II. Series.

$\begin{array}{llll}\text { QA273.B867 } & 1987 & 519.2 & 87-9422\end{array}$

(C) 1988 Springer Science+Business Media New York

Originally published by Springer-Verlag New York, Inc. in 1988

Softcover reprint of the hardcover 1st edition 1988

All rights reserved. This work may not be translated or copied in whole or in part without the written permission of the publisher Springer-Science+Business Media, LLC, except for brief excerpts in connection with reviews or scholarly analysis. Use in connection with any form of information storage and retrieval, electronic adaptation, computer software, or by similar or dissimilar methodology now known or hereafter developed is forbidden.

The use of general descriptive names, trade names, trademarks, etc. in this publication, even if the former are not especially identified, is not to be taken as a sign that such names, as understood by the Trade Marks and Merchandise Marks Act, may accordingly be used freely by anyone.

Typeset by Asco Trade Typesetting Ltd., Hong Kong.

9876543 (Corrected third printing, 1997)

SPIN 10643494

ISBN 978-1-4612-6996-0

ISBN 978-1-4612-1046-7 (eBook)

DOI 10.1007/978-1-4612-1046-7 
To my father 


\section{Preface}

The present textbook provides prerequisite material for courses in Physics, Electrical Engineering, Operations Research, and other fields of applied science where probabilistic models are used intensively. The emphasis has therefore been placed on modeling and computation.

There are two levels of modeling: abstráct and concrete.

The abstract level is relative to the axiomatization of Probability and provides a general framework that features an archetype of all concrete models, where the basic objects (events, probability, random variables), the basic concepts (independence, expectation), and the basic rule (countable additivity of probability) are given in abstract form. This moderately small axiomatic equipment, establishing Probability as a mathematical theory, suffices to produce a theorem called the strong law of large numbers that says in particular that in tossing coins "the average number of heads tends to $\frac{1}{2}$ as the number of independent tosses tends to infinity, if the coin is fair." This result shows that the axioms of probability are consistent with empirical evidence. (From a mathematical point of view, this a posteriori check of the relevance of the axioms is not necessary, whereas from the point of view of the modeler, it is of course of paramount importance.)

In the present book, the abstract framework is immediately introduced and a number of examples showing how this framework relates to the daily concerns of physicists and engineers is provided. The strong law of large numbers where the abstract framework culminates is proved in the last chapter.

The other level of modeling consists of fitting a given situation into the conceptual framework of the axiomatic theory when it is believed that random phenomena occur. This is a difficult exercise at the beginning, and the art of modeling can be acquired only through examples. Supplementary readings- 
entitled Illustrations - provide examples in which probabilistic models have been successfully developed.

They include, in particular, topics in stochastic processes and statistics as shown in the following list:

1. A Simple Model in Genetics: Mendel's Law and Hardy-Weinberg's Theorem

2. The Art of Counting: The Ballot Problem and the Reflection Principle

3. Bertrand's Paradox

4. An Introduction to Population Theory: Galton-Watson's Branching Process

5. Shannon's Source Coding Theorem: An Introduction to Information Theory

6. Buffon's Needle: A Problem in Random Geometry

7. An Introduction to Bayesian Decision Theory: Tests of Gaussian Hypotheses

8. A Statistical Procedure: The Chi-Square Test

9. Introduction to Signal Theory: Filtering.

The first chapter introduces the basic definitions and concepts of probability, independence, and cumulative distribution functions. It gives the elementary theory of conditioning (Bayes' formulas), and presents finite models, where computation of probability amounts to counting the elements of a given set. The second chapter is devoted to discrete random variables and to the generating functions of integer-valued random variables, whereas the third chapter treats the case of random vectors admitting a probability density. The last paragraph of the third chapter shows how Measure and Integration Theory can be useful to Probability Theory. It is of course just a brief summary of material far beyond the scope of an introduction to probability, emphasizing a useful technical tool: the Lebesgue convergence theorems. The fourth chapter treats two topics of special interest to engineers, operations researchers, and physicists: the Gaussian vectors and the Poisson process, which are the building blocks of a large number of probabilistic models. The treatment of Gaussian vectors is elementary but nevertheless contains the proof of the stability of the Gaussian character by extended linear transformations (linear transformations followed by passage to the limit in the quadratic mean). The Gaussian vectors and the Poisson process also constitute a source of examples of application of the formula of transformation of probability densities by smooth transformations of random vectors, which is given in the first paragraph and provides unity for this chapter. The last chapter treats the various concepts of convergence: in probability, almost sure, in distribution, and in the quadratic mean.

About 120 exercises with detailed solutions are presented in the main text to help the reader acquire computational skills and 28 additional exercises with outlines of solutions are given at the end of the book.

The material of the present textbook can be covered in a one-semester 
undergraduate course and the level can be adjusted simply by including or discarding portions of the last chapter, more technical, on convergences. The mathematical background consists of elementary calculus (series, Riemann integrals) and elementary linear algebra (matrices) as required of students in Physics and Engineering departments.

Gif-sur-Yvette, France

Pierre Brémaud 


\section{Contents}

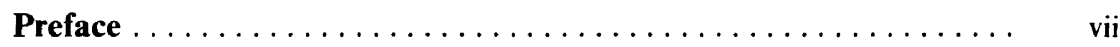

Abbreviations and Notations $\ldots \ldots \ldots \ldots \ldots \ldots \ldots \ldots \ldots \ldots \ldots \ldots$

\section{CHAPTER 1}

Basic Concepts and Elementary Models . . . . . . . . . . . . . 1

1. The Vocabulary of Probability Theory ................ 1

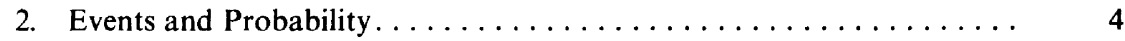

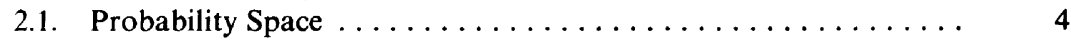

2.2. Two Elementary Probabilistic Models............... 7

3. Random Variables and Their Distributions ............... 9

3.1. Random Variables............................ 9

3.2. Cumulative Distribution Function. . . . . . . . . . . . 9

4. Conditional Probability and Independence. . . . . . . . . . . 12

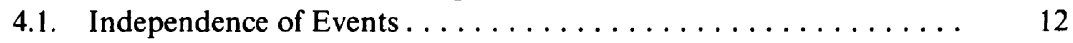

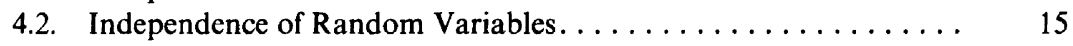

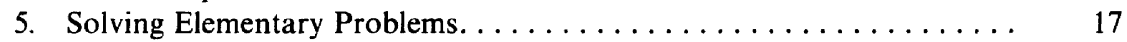

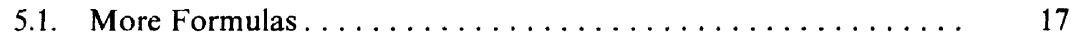

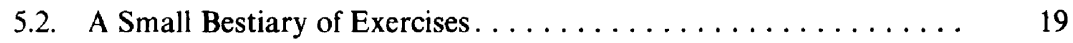

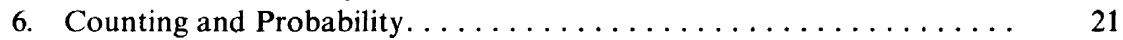

7. Concrete Probability Spaces. . . . . . . . . . . . . . . 26

Illustration 1. A Simple Model in Genetics: Mendel's Law and
Hardy-Weinberg's Theorem .............. 31

Illustration 2. The Art of Counting: The Ballot Problem and the Reflection
Principle $\ldots \ldots \ldots \ldots \ldots \ldots \ldots \ldots \ldots \ldots \ldots \ldots \ldots \ldots \ldots$

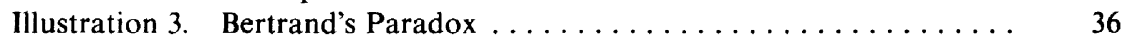

CHAPTER 2

Discrete Probability $\ldots \ldots \ldots \ldots \ldots \ldots \ldots \ldots \ldots \ldots \ldots \ldots \ldots \ldots \ldots$

1. Discrete Random Elements .................. 46 
1.1. Discrete Probability Distributions . . . . . . . . . . . . . 46

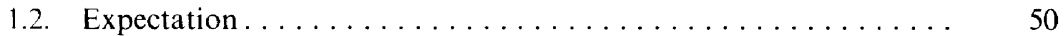

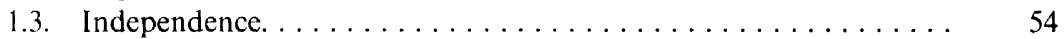

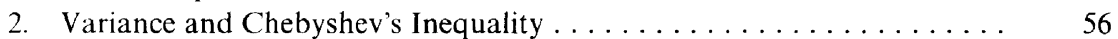

2.1. Mean and Variance. . . . . . . . . . . . . . . . . . . . . 56

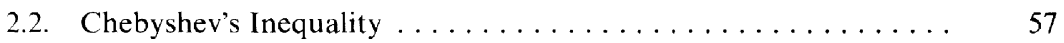

3. Generating Functions. . . . . . . . . . . . . . . . . 59

3.1. Definition and Basic Properties . . . . . . . . . . . . . 59

3.2. Independence and Product of Generating Functions ........ 62

Illustration 4. An Introduction to Population Theory: Galton-Watson's

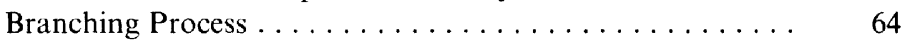

Illustration 5. Shannon's Source Coding Theorem: An Introduction to

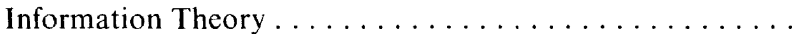

CHAPTER 3

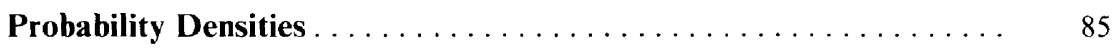

1. Expectation of Random Variables with a Density ........... 85

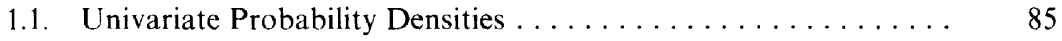

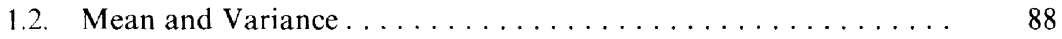

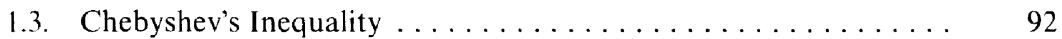

1.4. Characteristic Function of a Random Variable . . . . . . . . . . . 94

2. Expectation of Functionals of Random Vectors . . . . . . . . . . 96

2.1. Multivariate Probability Densities.................. 96

2.2. Covariance, Cross-Covariance, and Correlation. . . . . . . . . . 99

2.3. Characteristic Function of a Random Vector. . . . . . . . . . . . . 104

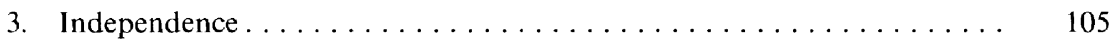

3.1. Independent Random Variables. . . . . . . . . . . . . . . 105

3.2. Independent Random Vectors . . . . . . . . . . . . . . 108

4. Random Variables That Are Not Discrete and Do Not Have a p.d. . . . 109

4.1. The Abstract Definition of Expectation . . . . . . . . . . 110

4.2. Lebesgue's Theorems and Applications .............. 114

Illustration 6. Buffon's Needle: A Problem in Random Geometry. . . . . . . 117

\section{CHAPTER 4}

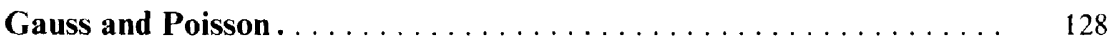

1. Smooth Change of Variables . . . . . . . . . . . . . . . . 128

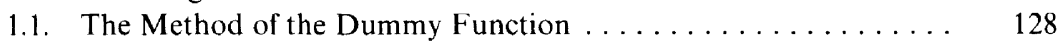

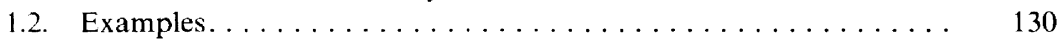

2. Gaussian Vectors .......................... 132

2.1. Characteristic Function of Gaussian Vectors . . . . . . . . . . 132

2.2. Probability Density of a Nondegenerate Gaussian Vector ...... 134

2.3. Moments of a Centered Gaussian Vector.............. 136

2.4. Random Variables Related to Gaussian Vectors . . . . . . . . . . 137

3. Poisson Processes. . . . . . . . . . . . . . . . . . . . . 140

3.1. Homogeneous Poisson Processes Over the Positive Half Line .... 140

3.2. Nonhomogeneous Poisson Processes Over the Positive Half Line . 142

3.3. Homogeneous Poisson Processes on the Plane . . . . . . . . . . 144

4. Gaussian Stochastic Processes . . . . . . . . . . . . . . . . 146

4.1. Stochastic Processes and Their Law.................. 146 
4.2. Gaussian Stochastic Processes . . . . . . . . . . . . . . . 147

Illustration 7. An Introduction to Bayesian Decision Theory: Tests of

Gaussian Hypotheses. . . . . . . . . . . . . . . . 148

\section{CHAPTER 5}

Convergences

1. Almost-Sure Convergence . . . . . . . . . . . . . . . . . . . 163

1.1. The Borel-Cantelli Lemma .................... 163

1.2. A Criterion for Almost-Sure Convergence . . . . . . . . . . . . 165

1.3. The Strong Law of Large Numbers . . . . . . . . . . . . . 166

2. Convergence in Law ........................... 168

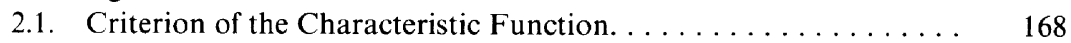

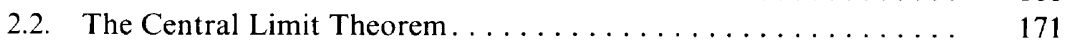

3. The Hierarchy of Convergences . . . . . . . . . . . . . 175

3.1. Almost-Sure Convergence Versus Convergence in Probability... 175

3.2. Convergence in the Quadratic Mean . . . . . . . . . . . . 176

3.3. Convergence in Law in the Hierarchy of Convergences . . . . . . . . 178

3.4. The Hierarchical Tableau . . . . . . . . . . . . . . . . 178

Illustration 8. A Statistical Procedure: The Chi-Square Test . . . . . . . . . 180

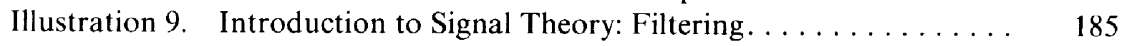

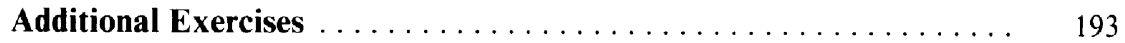

Solutions to Additional Exercises . . . . . . . . . . . . . . . . . . . 199

Index . . . . . . . . . . . . . . . . . . . . . 205 


\section{Abbreviations and Notations}

\section{Abbreviations}

$\begin{array}{ll}\text { a.s. } & \text { almost surely } \\ \text { c.d.f. } & \text { cumulative distribution function } \\ \text { c.f. } & \text { characteristic function } \\ \text { i.i.d. } & \text { independent and identically distributed } \\ \text { p.d. } & \text { probability density } \\ \text { q.m. } & \text { quadratic mean } \\ \text { r.v. } & \text { random variable }\end{array}$

\section{Notations}

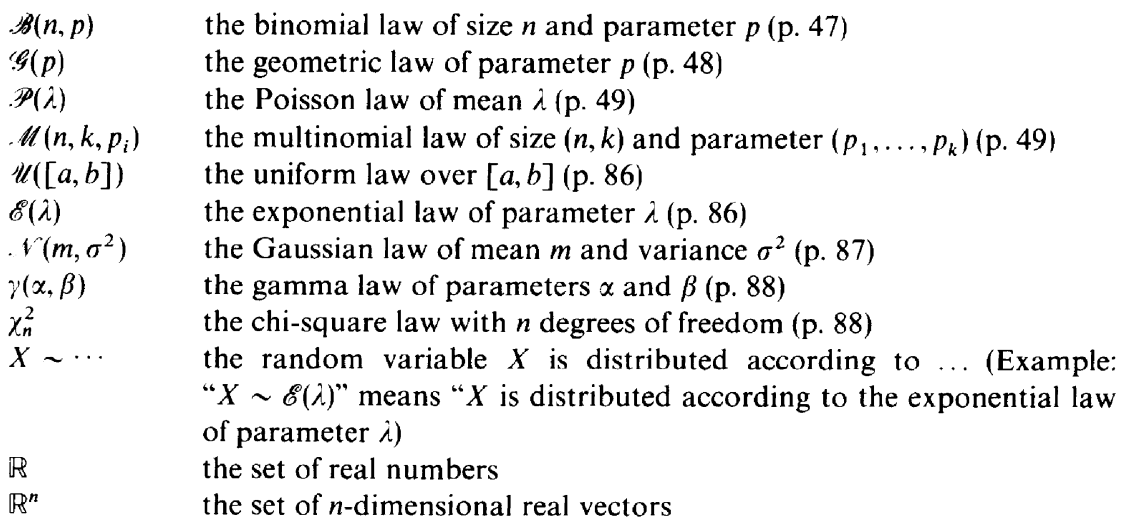


the Borelian sets of $\mathbb{R}^{n}$, that is: the smallest $\sigma$-field on $\mathbb{R}^{n}$ containing all the $n$-dimensional "rectangles"

$\mathbb{N} \quad$ the set of non-negative integers

$A^{\prime} \quad$ transpose of the matrix $A$

$u^{\prime}$

line vector, transpose of the column vector $u$ 